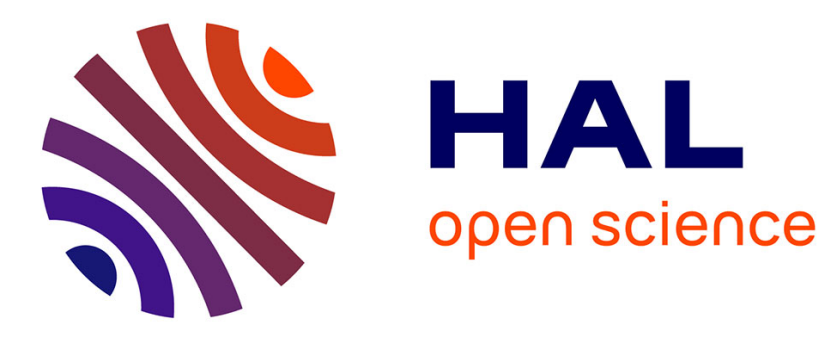

\title{
Instabilities in high power fiber lasers induced by stimulated Brillouin scattering
}

Djouher Mallek, Abdelhamid Kellou, Hervé Leblond, François Sanchez

\section{To cite this version:}

Djouher Mallek, Abdelhamid Kellou, Hervé Leblond, François Sanchez. Instabilities in high power fiber lasers induced by stimulated Brillouin scattering. Optics Communications, 2013, 308, pp.130-135. 10.1016/j.optcom.2013.06.062 . hal-03204348

\section{HAL Id: hal-03204348 \\ https://univ-angers.hal.science/hal-03204348}

Submitted on 21 Apr 2021

HAL is a multi-disciplinary open access archive for the deposit and dissemination of scientific research documents, whether they are published or not. The documents may come from teaching and research institutions in France or abroad, or from public or private research centers.
L'archive ouverte pluridisciplinaire HAL, est destinée au dépôt et à la diffusion de documents scientifiques de niveau recherche, publiés ou non, émanant des établissements d'enseignement et de recherche français ou étrangers, des laboratoires publics ou privés. 


\title{
Instabilities in high power fiber lasers induced by stimulated Brillouin scattering
}

\author{
Djouher Mallek $^{\mathrm{a}, \mathrm{b}, *}$, Abdelhamid Kellou ${ }^{\mathrm{a}}$, Hervé Leblond ${ }^{\mathrm{b}}$, François Sanchez ${ }^{\mathrm{b}}$ \\ a Laboratoire d'Electronique Quantique, Faculté de Physique, Université des Sciences et de la Technologie Houari Boumediene, \\ B.P. 32, El-Alia Bab-Ezzouar 16111, Alger, Algeria \\ ${ }^{\mathrm{b}}$ LUNAM Université, Université d'Angers, Laboratoire de Photonique d'Angers LPhiA, E.A. 4464, 2 Bd Lavoisier, 49000 Angers, France
}

\section{A R T I C L E I N F O}

\section{Article history:}

Received 9 December 2012

Received in revised form

27 June 2013

Accepted 30 June 2013

Available online 11 July 2013

Keywords:

Brillouin scattering

Instabilities

Quasi-periodic

Chaotic

High power fiber lasers

\begin{abstract}
A B S T R A C T
We investigate numerically different types of instabilities in a high loss Fabry-Perot laser cavity in presence of the stimulated Brillouin scattering. Our results reveal many interesting dynamical behaviors such as periodic, quasi-periodic and chaotic.
\end{abstract}

(c) 2013 Elsevier B.V. All rights reserved.

\section{Introduction}

Brillouin instabilities have attracted much attention since the early stage of single-mode fiber realization. Indeed, the instabilities observed when an optical wave travels a single mode passive fiber have been attributed to the presence of the Brillouin backscattering defined as a coupling between optical and acoustic waves. The acoustic wave produces a moving index grating in which the pump beam is back-reflected. The backscattered Stokes wave is frequency down-shifted. A chaotic behavior is observed very close to the Brillouin threshold without any optical feedback [1]. The external feedback, provided by the fiber ends, leads to additional dynamical behaviors such as periodic and quasi periodic oscillations [1-3].

Various theoretical studies were made to explain the emergence of the chaos and its suppression. The interaction of the nonlinear refraction with the Brillouin scattering is identified as being responsible for various dynamical behaviors [4]. Moreover, the existence of the chaos without any feedback is related to the stochastic nature of the spontaneous Brillouin scattering $[5,6]$. The feedback suppresses the stochastic nature of the Brillouin

\footnotetext{
*Corresponding author at: Laboratoire d'Electronique Quantique, Faculté de Physique, Université des Sciences et de la Technologie Houari Boumediene, B.P. 32, El-Alia Bab-Ezzouar 16111, Alger, Algeria. Tel.: +213 21247344; fax: +213 21247344.

E-mail addresses: djouher.bouras@etud.univ-angers.fr, djouher.mallek@umbb.dz (D. Mallek).
}

effect allowing a purely deterministic chaotic behavior resulting from the interplay between Brillouin effect and nonlinear refraction $[3,6]$.

The Brillouin effect has been also studied in fiber ring resonators in order to realize Brillouin fiber lasers [7,8]. Various instabilities were observed in such optical configurations. As demonstrated in reference [8], the nonlinear refraction is an essential mechanism in the destabilization of the dynamical behavior.

On the other hand, ytterbium-doped double clad fiber lasers exhibit temporal instabilities which were attributed to Brillouin scattering as demonstrated in [9] where the laser was stabilized in a unidirectional cavity thus avoiding any backscattered waves. Brillouin Stokes waves have been experimentally evidenced in [10]. Further studies concerned the influence of the cavity losses on the operating regime. It was demonstrated that a low losses cavity favours continuous operating regime while the high losses configuration leads to a self-pulsing instability [11]. Surprisingly, there are few papers concerning the theoretical modeling of a fiber laser in the presence of Brillouin effect. The first one is related to the influence of the Brillouin effect on the pulse shape in an actively Q-switch ytterbium-doped double-clad fiber laser [12]. In the previous work, we have proposed a simple model able to describe the low-frequency self-pulsing instability [13] thus demonstrating that this instability is a direct consequence of the stimulated Brillouin scattering without any active or passive forced modulation. The model was based on 4 intensity equations (forward and backward components for laser and Stokes waves). 
In this first approach, we neglected the acoustic wave equation thus implying an instantaneous Brillouin gain. This approximation limited the validity of the model to the description of phenomena on a time scale well above the phonon lifetime. We considered a Fabry-Perot cavity inside which the spatial hole burning occurs. As a consequence, different wavelengths experienced gain from different dipoles distributions thus resulting in the existence of different classes of dipoles [14]. Consequently, the model in [13] assumes two distinct population inversions, one associated with the laser field and the other to the Stokes wave. Both are coupled through cross-saturation. The constructed model allowed demonstrating that the Brillouin back-scattering can induce a self-pulsing with a large period compared to the round trip time in the high losses cavity case. For the low losses cavity, the laser remains stable over a large range of pumping powers [13]. Before proceeding it is worth to discuss about the strength of the cross-saturation between laser and Brilllouin waves. The cross-saturation parameter $\beta$ is generally of the order of $0.5[15,16]$. However with such conventional value, no instability occurs and only the Brillouin wave oscillates, the laser field vanishes. This is due to the fact that the coupling between the two waves is too strong. The coupling between the two waves has two origins; the first one is the crosssaturation and the second one is the direct Brillouin coupling. The latter is fixed by the host material. As a consequence we decided in [13] to reduce the value of the cross-saturation which was therefore considered as a free parameter. The instability of the solution appeared for very low value of $\beta$ of the order of 0.05 . This value allowed us to obtain a self-pulsing instability without need to introduce a saturable absorber [12]. Also the value of $\beta$ appears unrealistic it is necessary to obtain results in good agreement with the experimental data $[10,11,17]$. At this stage of our knowledge the low value of $\beta$ is not physically justified.

In this paper, the influence of Brillouin effect on the dynamics of high power fiber lasers is described with an improved model that takes into account the dynamics of the acoustic wave. Such approach leads to results in better agreement with the experimental ones reported in $[10,11]$ and also to new dynamical behaviors. In particular, the dynamics of the acoustic wave will allow to (1) find an instability threshold close to that observed experimentally [10], (2) reach a self-pulsing regime occurring on the nanosecond range as for experimental observations [17] and (3) identify new regimes which were not found by using the intensity model [13]. An extensive study has been performed for the identification of these regimes by using several analysis tools such as phase portraits, first returns map and Fourier spectra. This paper is organized as follows. Section 2 is devoted to the theoretical model. Numerical simulations are presented in Section 3. Several dynamical behaviors are identified.

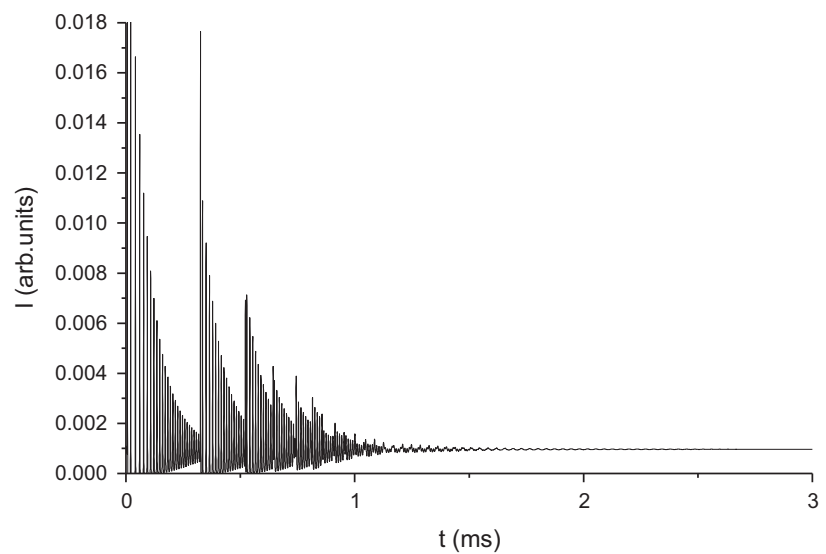

Fig. 1. Temporal evolution of the total output intensity for a pumping ratio $r=1.5$.

\section{The model}

We investigate numerically an ytterbium doped fiber laser, with several watt of output power [17]. The laser is pumped at $976 \mathrm{~nm}$ and it emits at $1080 \mathrm{~nm}$, it is considered as a quasi-three levels system. We consider a Fabry-Perot fiber laser cavity and assume that the fiber supports a forward and a backward laser waves which are modeled through their amplitudes. For simplicity we assume that there is only the first order Stokes wave with its forward and backward components. In contrast with [13], we include the equation for the acoustic wave. Optical Kerr effect is neglected in this paper although it can be of importance in special situations. What we gain with our new approach is that the results are not limited by the phonon lifetime and that the model is valid in a timescale below this lifetime. The different waves are described in terms of their amplitudes which are subjected to the classical boundary conditions on the mirrors. The forward (backward) component of the laser wave is coupled to the backward (forward) component of the Stokes wave as a result of the Brillouin coupling. In addition, all waves fall below the gain curve and consequently are coupled through the gain medium, at this stage we have two possibilities. The first one is to assume that all the fields are amplified through stimulated emission associated to a unique population inversion. This approach, used in [12], does not seem satisfactory because the spatial hole burning in standingwave lasers yields naturally to the existence of different classes of

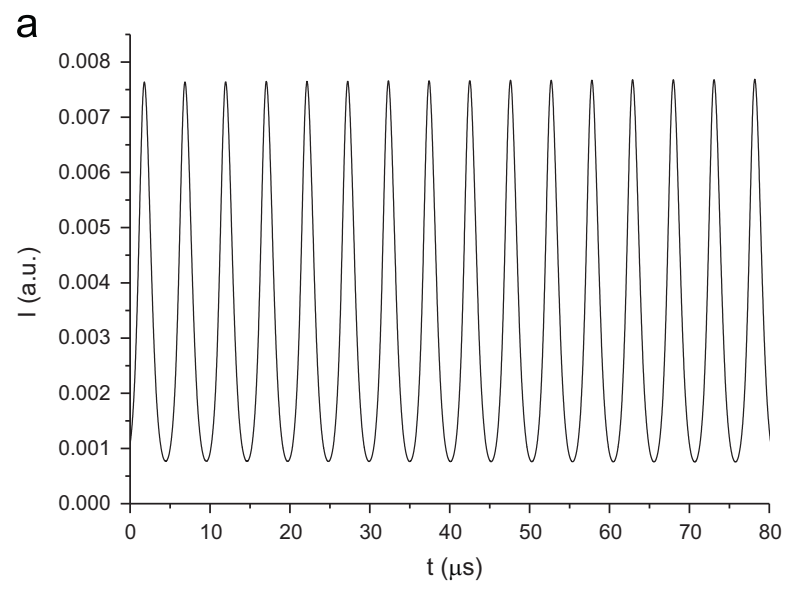

b

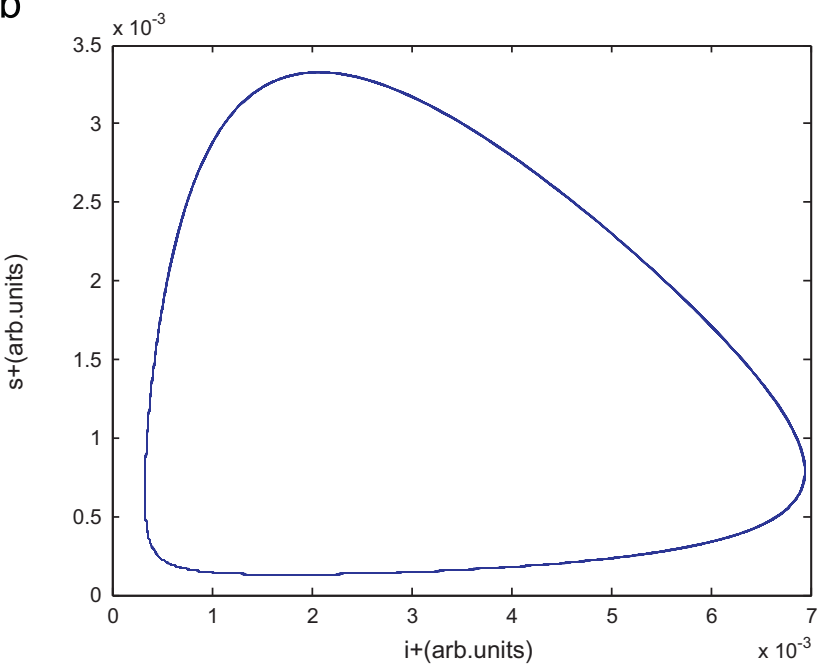

Fig. 2. (a) Temporal evolution of the total output intensity for $r=2.3$ and (b) corresponding phase diagram in the plane $\left(i^{+}, s^{+}\right)$. 
dipoles [14]. The second approach, that we have decided to use, is a consequence of the spatial hole burning and consists to consider two classes of atoms, each associated to one wavelength [14]. Such approach has been used successfully for the description of the dynamics of dual-wavelength erbium-doped fiber lasers [16]. Of course, cross-saturation occurs between the laser and the stokes waves.

In its final form, the model involves 2 material equations (population inversions) and 6 field equations (forward and backward components of the laser, the Stokes and the acoustic waves). The normalized equations write as:

$\frac{\partial d_{1}}{\partial t}=p-a_{1}\left(1+d_{1}\right)-2 d_{1}\left[\left(\left|e_{p}^{+}\right|^{2}+\left|e_{p}^{-}\right|^{2}\right)+\beta\left(\left|e_{s}^{+}\right|^{2}+\left|e_{s}^{-}\right|^{2}\right)\right]$,

$\frac{\partial d_{2}}{\partial t}=\gamma p-a_{1}\left(1+d_{2}\right)-2 d_{2}\left[\left(\left|e_{s}^{+}\right|^{2}+\left|e_{s}^{-}\right|^{2}\right)+\beta\left(\left|e_{p}^{+}\right|^{2}+\left|e_{p}^{-}\right|^{2}\right)\right]$,

$\frac{\partial e_{p}^{ \pm}}{\partial t} \pm \frac{\partial e_{p}^{ \pm}}{\partial \zeta}=-\frac{1}{2} G e_{s}^{\mp} e_{a}^{ \pm}-\frac{1}{2} \alpha_{c} e_{p}^{ \pm}+\frac{1}{2} A\left(d_{1}+\beta d_{2}\right) e_{p}^{ \pm}$,

$\frac{\partial e_{s}^{ \pm}}{\partial t} \pm \frac{\partial e_{s}^{ \pm}}{\partial \zeta}=\frac{1}{2} G e_{p}^{\mp} e_{a}^{\mp *}-\frac{1}{2} \alpha_{c} e_{s}^{ \pm}+\frac{1}{2} A\left(d_{2}+\beta d_{1}\right) e_{s}^{ \pm}$,

$\frac{\partial e_{a}^{ \pm}}{\partial t}=\frac{1}{2} \alpha_{a}\left(e_{p}^{ \pm} e_{s}^{\mp *}-e_{a}^{ \pm}\right)$,

with the boundary conditions on the mirrors

$e_{p}^{+}(0, t)=\sqrt{R_{1}} e_{p}^{-}(0, t)$

$e_{p}^{-}(\ell, t)=\sqrt{R_{2}} e_{p}^{+}(\ell, t)$

$e_{s}^{+}(0, t)=\sqrt{R_{1}} e_{s}^{-}(0, t)$
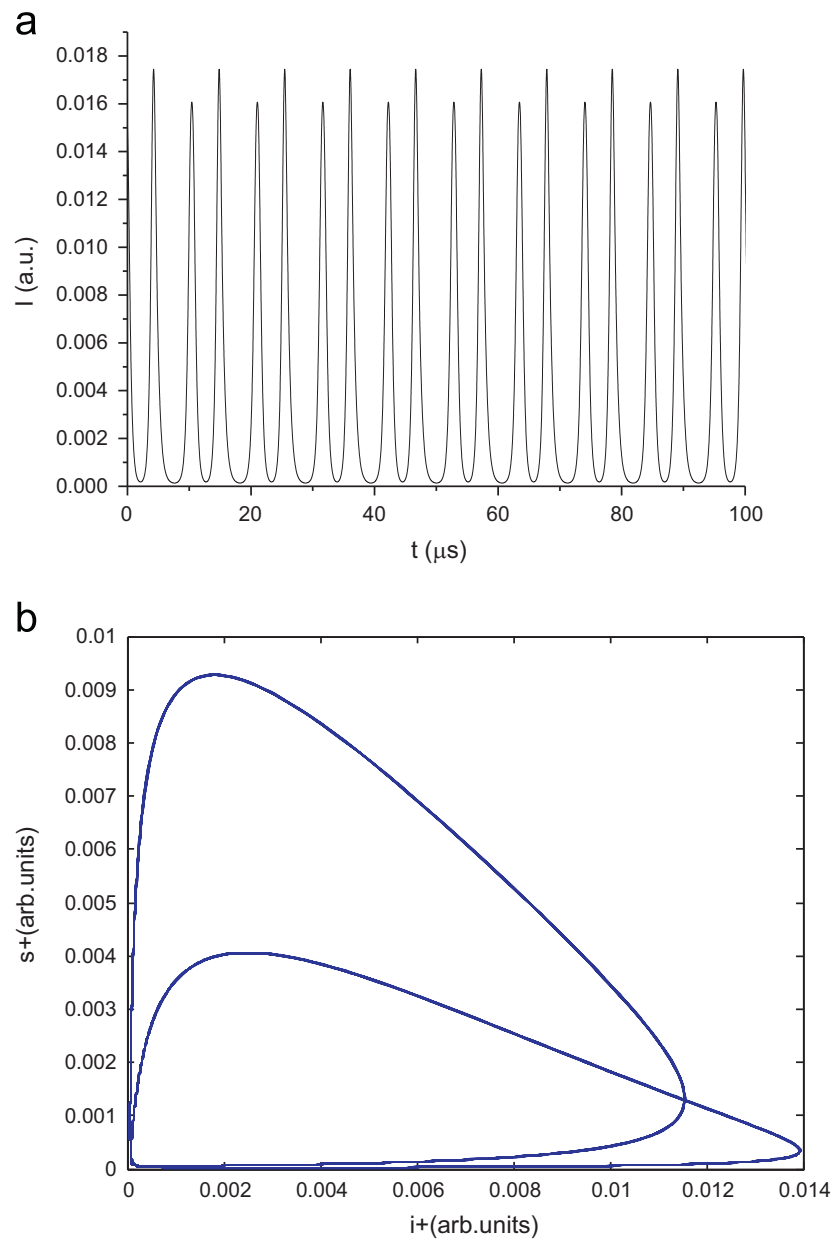

Fig. 3. (a) Temporal evolution of the total output intensity for $r=2.5$ and (b) corresponding phase diagram in the plane $\left(i^{+}, s^{+}\right)$. $e_{s}^{-}(\ell, t)=\sqrt{R_{2}} e_{s}^{+}(\ell, t)$

The $d_{n}$ 's are the population inversions normalized versus the concentration of active ions $\left(N_{0}=10^{24} \mathrm{~m}^{-3}\right) \cdot e_{p}^{ \pm}, e_{s}^{ \pm}$and $e_{a}^{ \pm}$are the normalized laser, Stokes and acoustic field amplitudes. The superscript plus and minus stand for forward and backward components, respectively. The time $t$ is normalized versus the photon transit time along the fiber $\left(T_{r}=48 \mathrm{~ns}\right)$ and the longitudinal coordinate $\zeta=z / L$ is normalized versus the fiber length ( $L=10 \mathrm{~m}$ ) which is assumed to coincide with the total cavity length, so that the normalized cavity length is $\ell=1$. The other

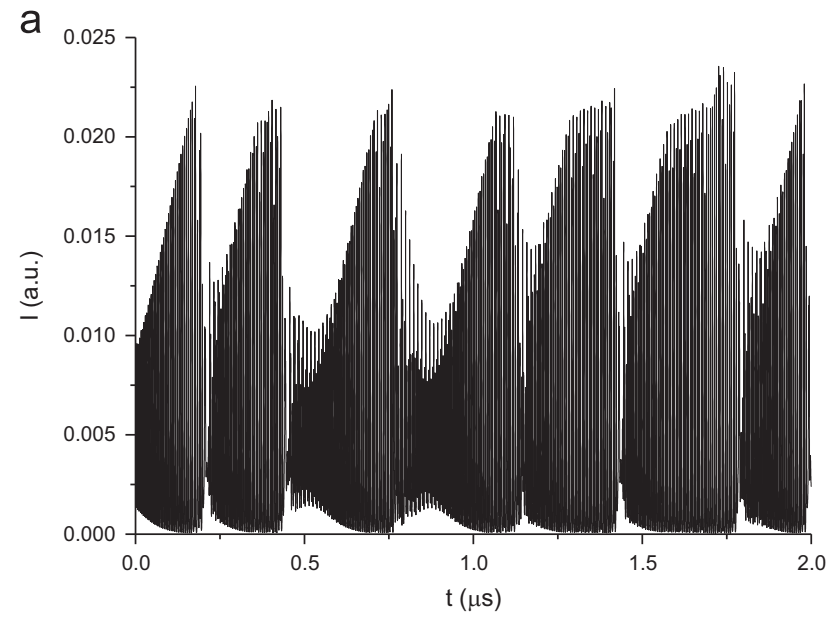

b

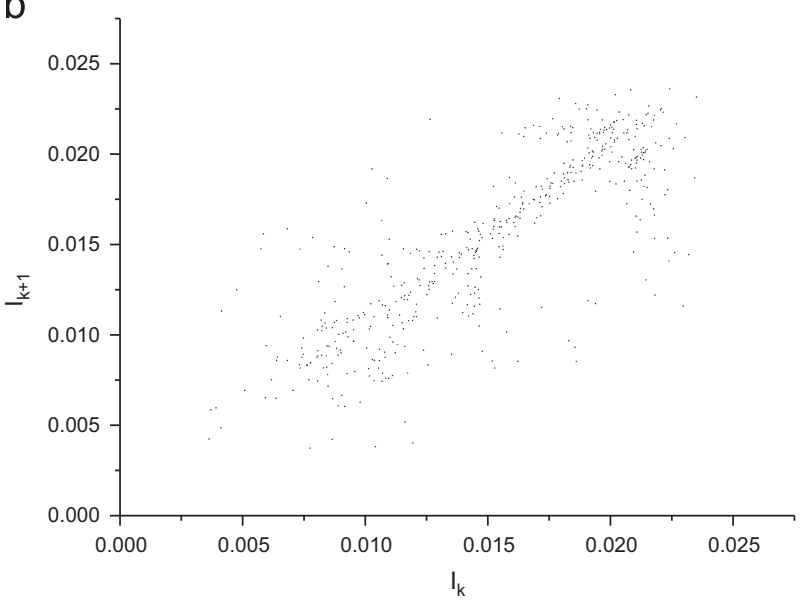

C

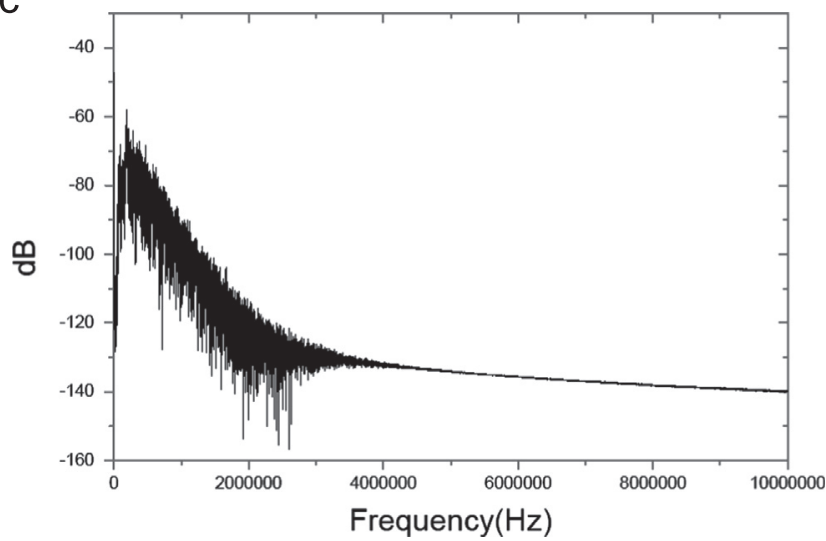

Fig. 4. (a) Temporal evolution of the total output intensity for $r=2.7$ and (b) corresponding first return map and (c) low frequency spectrum. 

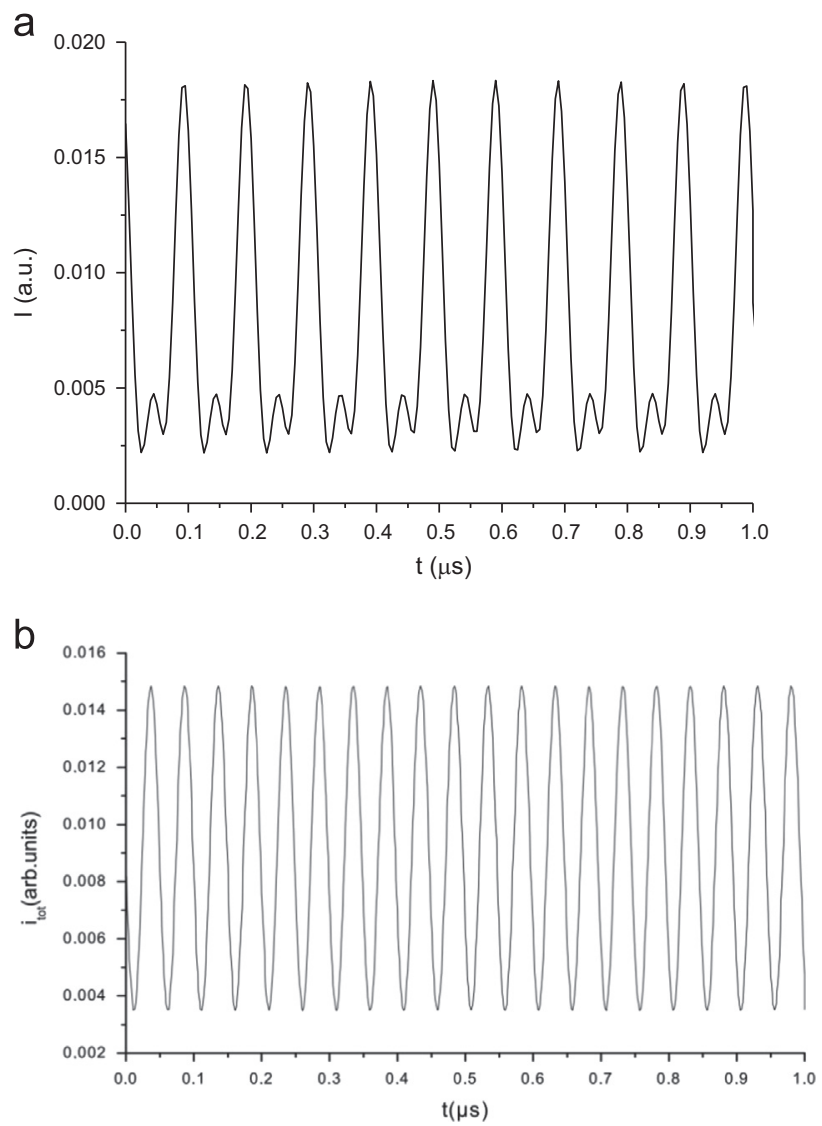

Fig. 5. Temporal evolution of the total output intensity. (a) periodic regime for $r=4$ and (b) period having observed for $r=4.5$.

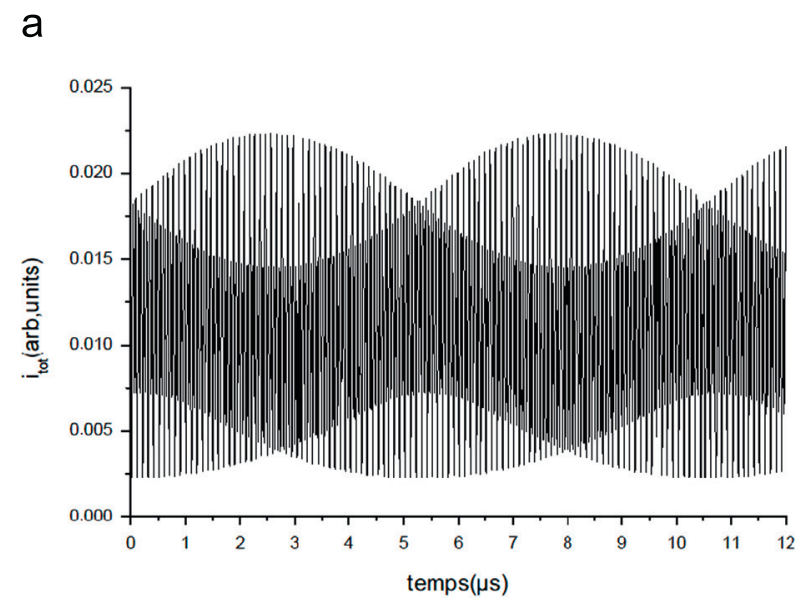

b

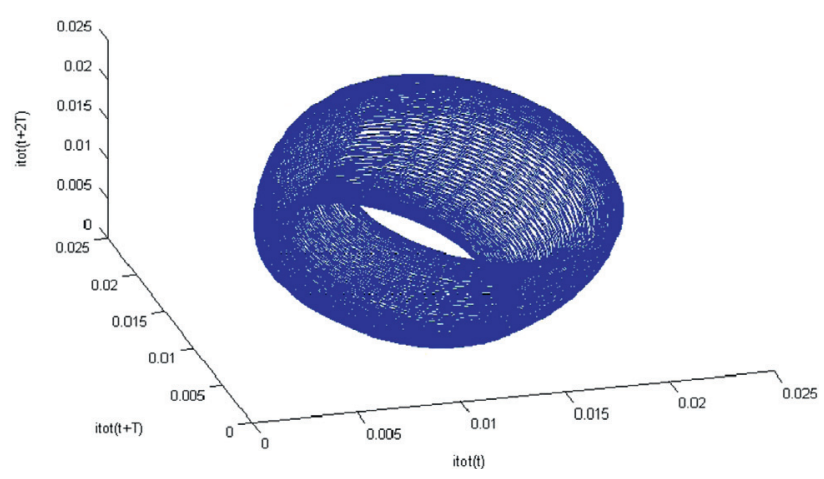

parameters are $a_{1}=T_{r} / \tau, A=\sigma N_{0} L, G=g_{B} L I_{0}, I_{0}=\hbar \omega /\left(\sigma T_{r}\right), \alpha_{c}=\alpha L$ and $\alpha_{a}=T_{r} \Gamma, \Gamma$ the damping coefficient for acoustic wave.

$\tau=800 \mu \mathrm{s}$ is the lifetime of the upper lasing level, $\sigma=32 \times$ $10^{-25} \mathrm{~m}^{2}$ the emission cross-section, $g_{B}$ the Brillouin gain and $\alpha=0.0458 \mathrm{~m}^{-1}$ the optical absorption coefficient of the fiber. The normalized Brillouin gain is $G=60$ as discussed in [13]. The normalized acoustical absorption coefficient is $\alpha_{a}=3 . \omega=2 \pi c / \lambda$ Is the optical pulsation with $\lambda=1.08 \mu \mathrm{m}$. $\mathrm{p}$ Is the pumping parameter and is assumed to be uniform along the fiber. $\gamma$ represents a dichroism in the pumping process in order to take into account some anisotropy of the laser $[15,16] . \beta$ is the crosssaturation parameter and physically represents the strength of the coupling between the laser field and the Stokes through the amplifying medium [14-16]. For the numerical simulations we will take $\gamma=0.7$ and $\beta=0.05$ [13]. $R_{1}$ and $R_{2}$ are the reflection coefficients of the mirrors.

The laser threshold can be easily determined from the system (1) [13]

$p_{t h}=\frac{a_{1}}{1+\beta \gamma}\left[1+\beta+\frac{1}{A}\left(\alpha_{c}+\frac{1}{2 \ell} \operatorname{Ln} \frac{1}{\sqrt{R_{1} R_{2}}}\right)\right]$

\section{Periodic, pseudo-periodic and chaotic regimes}

The above model of the coupled amplitudes is solved numerically by a fourth order Runge-Kutta algorithm for the temporal derivatives. The $z$ derivatives are computed by means of five-point finite differences. We are interested on the temporal evolution of the total output intensity of the laser defined as $I(\ell, t)=i^{+}(\ell, t)+$ $s^{+}(\ell, t)$ where $i^{+}(\ell, t)=R_{2}\left|e_{p}^{+}(\ell, t)\right|^{2}$ and $s^{+}(\ell, t)=R_{2}\left|e_{s}^{+}(\ell, t)\right|^{2}$ are the output intensities of the forward component of the laser and
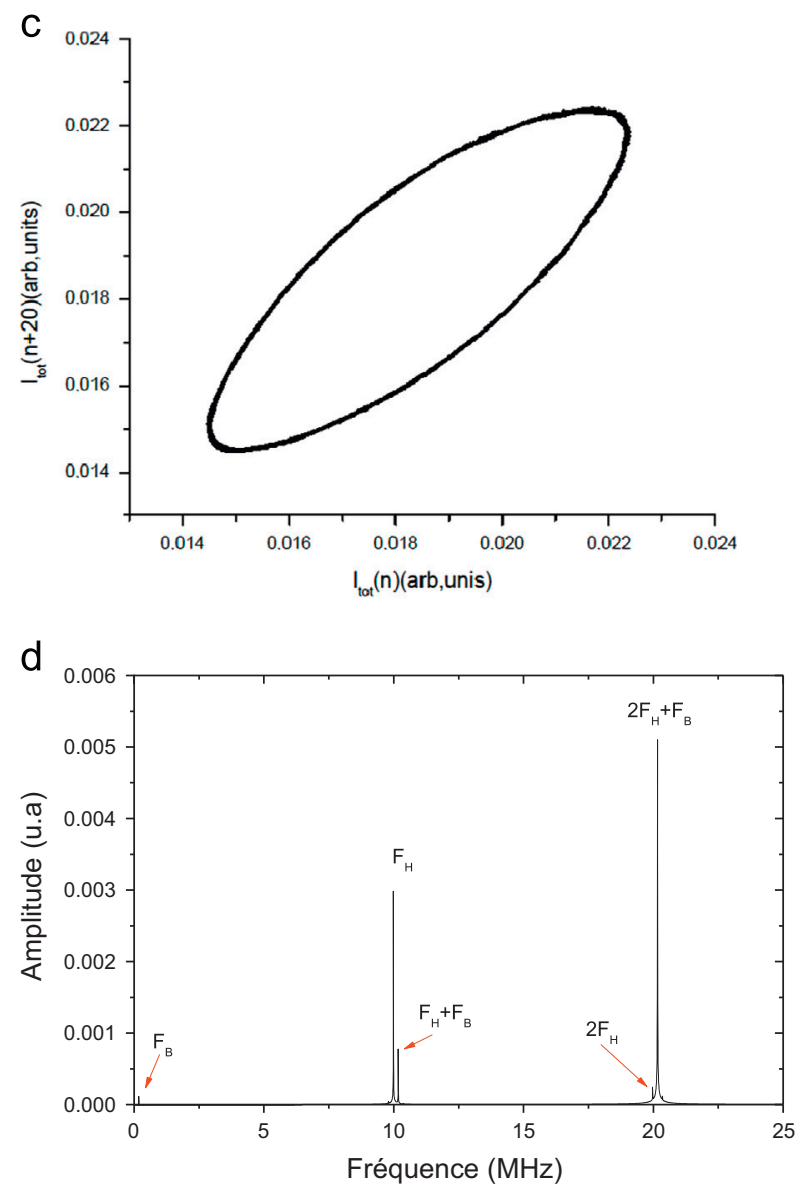

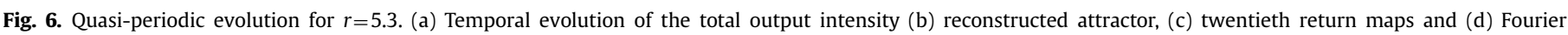
spectrum of the output intensity. 
the Stokes waves, respectively. The external control parameter is the pumping ratio defined as $r=p / p_{t h}$.

In [13] we showed that instabilities occurred only in high-loss cavity. Therefore in this paper we restrict our analysis to this case, assuming $R_{1}=1$ and $R_{2}=0.16$. In the following the pumping ratio is varied and the output intensity is analyzed. The numerical results show that the laser presents a continuous behavior for pumping rates $r<2.3$ as illustrated in Fig. 1 obtained for a pumping ratio $r=1.5$. One can see that after the transient, the laser operates in continuous regime (CW). The unusual transient oscillations are due to the coupling between the laser field and the Stokes wave. A regular self pulsing instability appears for $r=2.3$ with a period $T=5 \mu \mathrm{s}$ and a pulse duration of $2 \mu \mathrm{s}$, as shown in Fig. 2. The value of the instability threshold is considerably reduced with regard to the value obtained with the model of [13] $(r \approx 6,3)$, and is closer to the experimental value $r \approx 1.65$ [10]. The phase diagram shown in Fig. 2(b) illustrates the trajectory in the plane $\left(i^{+}, s^{+}\right)$. It is a limit cycle associated with a periodic regime. While the value of pumping increases, period doubling occurs, the temporal evolution of the intensity is $2 T$-periodic for
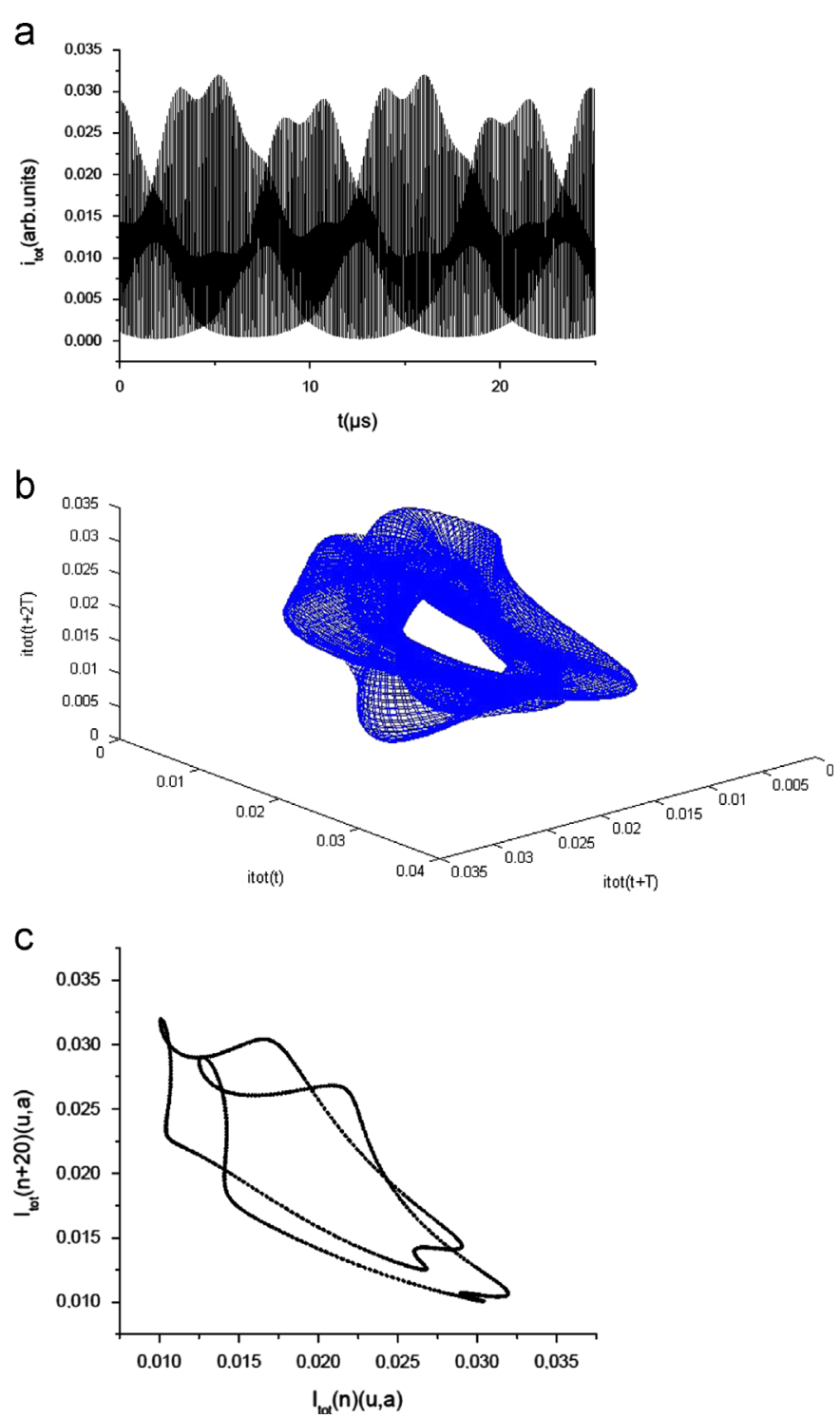

Fig. 7. Quasi-periodic evolution for $r=5$.7. (a) Temporal evolution of the total output intensity (b) reconstructed attractor showing a folded torus and (c) twentieth return maps. $r=2.5$, as shown in Fig. 3(a). The phase diagram of Fig. 3(b) confirms the period doubling since it exhibits a double loop [16].

By further increasing the pumping rate up to $r=2.7$, the output intensity presents several trains of irregular pulses as shown in Fig. 4(a). We note that between two successive trains, the amplitude of the pulses decreases considerably, and then increases gradually during the duration of the next train of pulses. The laser exhibits a fundamental period of about $5 \mu \mathrm{s}$ but this is not sufficient to fully characterize the dynamics since there is a slow evolution which seems to be random. To determine the nature of the dynamical behavior for $r=2.7$, we first draw the first return map constructed with the series of the maxima of the output intensity. The last one is the unidirectional application which relates the coordinate $I_{k+1}$ of a point with time $(k+1) T$ to the coordinate $I_{k}$ at time $k T$ [18]. This application can be considered as a reconstruction of the Poincare section by means of shifted coordinates $I_{k+1}=f\left(I_{k}\right)$. The first return map corresponding to the time evolution of Fig. 4(a) is given in (b). It exhibits an irregular distribution of points without any known pattern. Such first-return map is characteristic of a chaotic behavior $[18,19]$. The

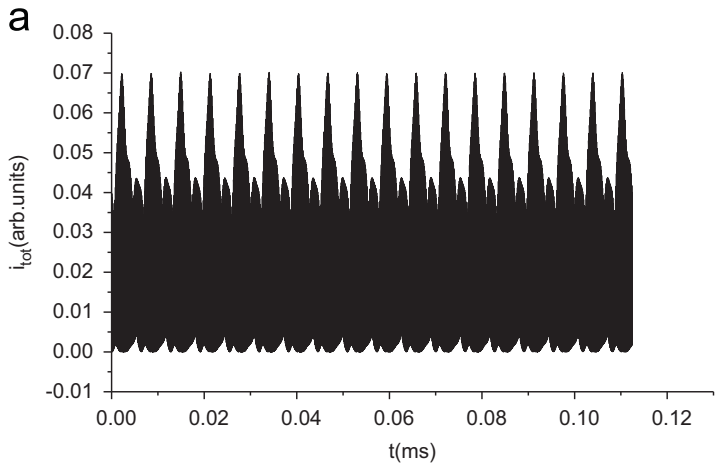

b
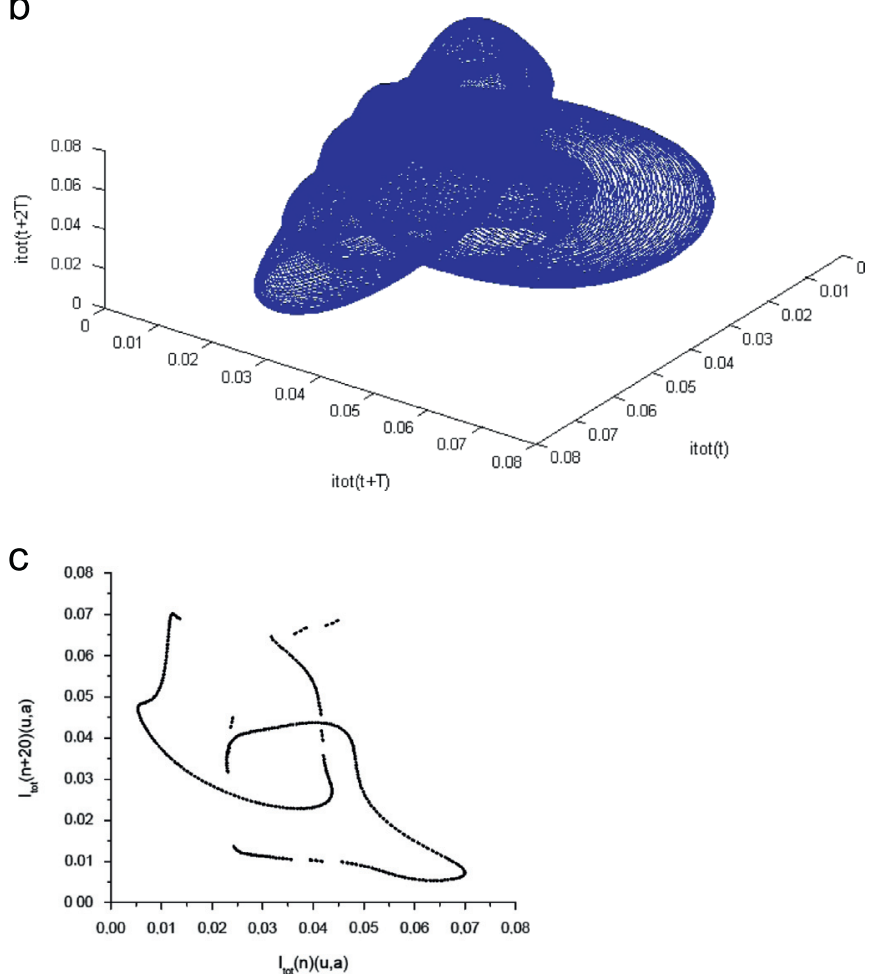

Fig. 8. Quasi-periodic evolution for $r=9$. (a) Temporal evolution of the total output intensity (b) reconstructed attractor showing a higher dimension torus and (c) twentieth return maps. 
corresponding low frequency spectrum given in Fig. 4(c) illustrates an important band of noise without any characteristic frequency thus confirming the chaotic regime. The dynamic stabilizes on a double loop periodic orbit for $r=4$. The period of the pulses is $T^{\prime}=$ $100 \mathrm{~ns}$ which corresponds to the round trip time of the cavity while the pulse duration is $18 \mathrm{~ns}$ which is close to the lifetime of the acoustic wave (Fig. 5(a)). For $r=4.5$, period halving occurs and a sinusoidal periodic regime is observed, with a period $T^{\prime} / 2=50 \mathrm{~ns}$ and a pulse duration of $18 \mathrm{~ns}$ as shown in Fig. 5(b).

The dynamics completely changes for a pumping rate $r=5.3$ up to $r=9$ for which a quasi-periodic regime appears. An example of time evolution is given in Fig. 6(a) which exhibits a fast evolution modulated with a very low frequency. The reconstructed attractor is shown in Fig. 6(b). It consists in a torus $T^{2}$ characteristic of quasi-periodicity. The reconstruction has been done using the delayed coordinates in a three-dimensional space [18]. The twentieth return map of Fig. 6(c) shows a closed curve confirming the quasi-periodic regime. The system is driven by two incommensurable frequencies which can be pointed out with the Fourier spectrum of the output intensity. The spectrum is given in Fig. 6 (d) and confirms two incommensurable frequencies $F_{L}$ (low frequency) and $F_{H}$ (high frequency) and linear combinations of these two fundamental frequencies. The low frequency $F_{L}=180 \mathrm{kHz}$ corresponds physically to the relaxation oscillation frequency while $F_{H}=9.985 \mathrm{MHz}$ is the free spectral range. The regime is slightly
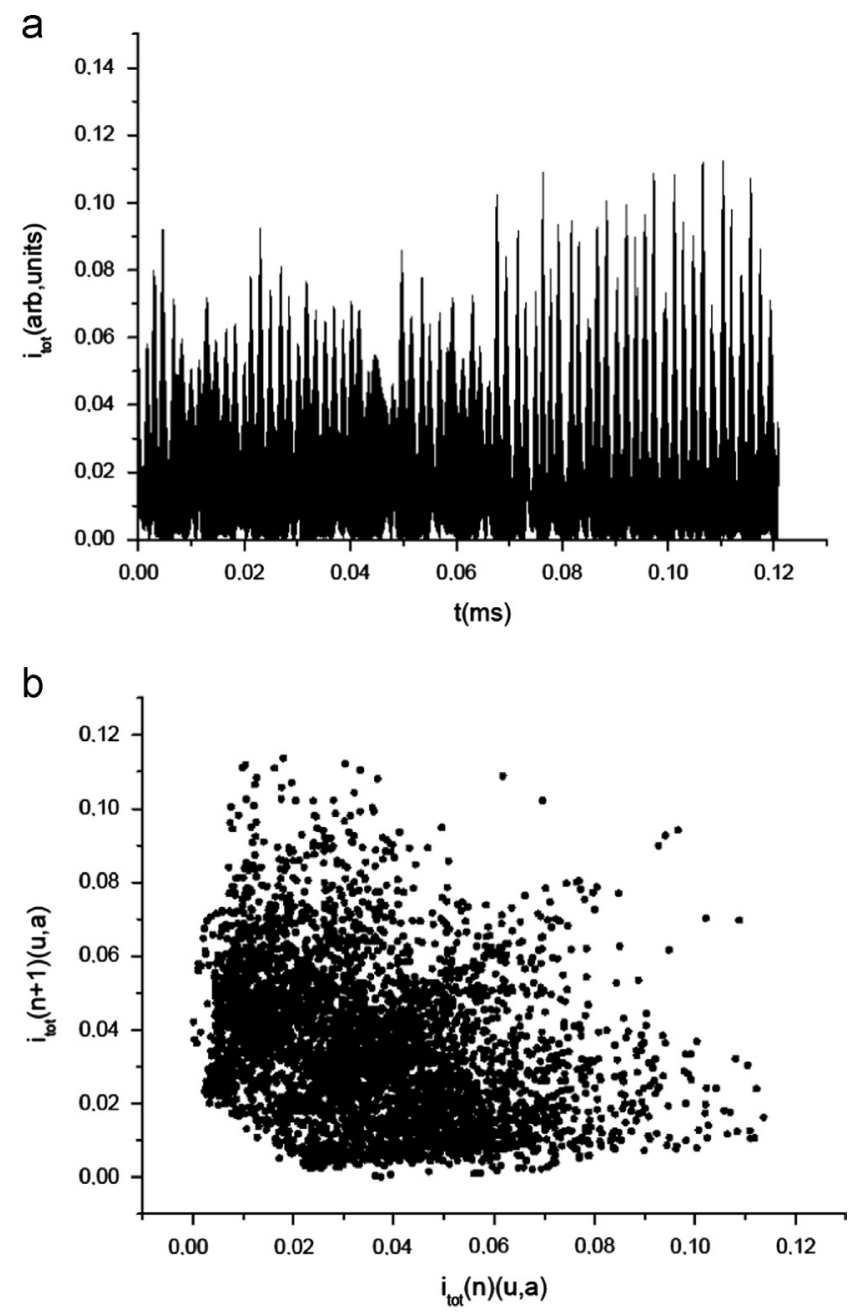

different for $r=5.7$ as shown in the temporal trace of Fig. 7(a). The diagram of the twentieth return map shows a kind of doubling (Fig.7 (c)) which reveals that the trajectory evolves on both parts of a folded torus (Fig. 7.b) due to the importance of the second harmonic $2 F_{L}$ of the low frequency [19]. Note that the subharmonic $F_{L} / 2$ (and combinations of the form $(n+1 / 2) F_{L}$ ) also appear in the low-frequency spectrum. The results for $r=9$ are given in Fig. 8 . The twentieth return map presents opened curves (Fig. 8(c)) thus confirming the quasiperiodic regime. However it reveals that the attractor is a not a mere torus $T^{2}$ (Fig. 8(b)) but a torus embedded into a higher dimensional space (at least four instead of three) [20]. For $r=9.003$ the systems falls into a chaotic dynamics confirmed with a first return map, which presents an irregular distribution of points (Fig. 9).

\section{Conclusion}

In summary we have investigated the dynamics of a high power continuously pumped fiber laser in presence of stimulated Brillouin scattering. The pumping ratio has been used as the external control parameter. From the dynamical point of view we observed first a route to chaos by period doubling, then a return to a sinusoidal regime by period halving, and then again a route to chaos, now through quasi-periodic process. Both the subharmonic and the quasi-periodic routes to chaos are wellknown typical processes. From the physical point of view we have pointed out two distinct self-pulsing regimes, one in the microsecond range and the other in the nanosecond range. These regimes were experimentally evidenced in the Yb-doped doubleclad fiber laser [17]. Our model is then in good qualitative agreement with the experiment and considerably improves the model which disregards the dynamics of the acoustic wave.

A better description of the dynamics could be obtained through bifurcation diagrams. However, this is not achievable, at least with our present numerical code, due to excessive computation time. Improvement of the numerical code can be envisaged.

\section{References}

[1] R.G. Harrison, J.S. Uppal, A. Johnstone, J.V. Moloney, Physical Review Letters 65 (167-1) (1990) 70.

[2] A. Johnstone, W. Lu, J.s. Uppal, R.G. Harrison, Optics Communications 81 (1991) 222. [3] D. Yu, W. Lu, R.G. Harrison, Physical Review A 51 (1995) 669.

[4] A.L. Gaeta, R.W. Boyd, Phys. Rev. A. 44 (1991) 3205.

[5] M. Damming, G. Zinner, F. Mitschke, H. Welling, Physical Review A 48 (1993) 3301.

[6] R.G. Harrison, P.M. Ripley, W. Lu, Physical Review A 49 (1994) 24.

[7] J. Botineau, C. Leycuras, C. Montes, E. Picholles, Journal of the Optical Society of America B 6 (3) (1989) 300.

[8] H. Li, K. Ogusu, Optical Review 7 (2000) P_303.

[9] A. Hideur, T. Chartier, C. Özkul, F. Sanchez, Optics Communications 186 (2000) 311.

[10] M. Salhi, A. Hideur, T. Chartier, M. Brunel, G. Martel, C. Özkul, F. Sanchez, Optics Letters 27 (2002) 1294.

[11] B. Ortaç, A. Hideur, T. Chartier, M. Brunel, G. Martel, C. Özkul, M. Salhi, F. Sanchez, Optics Communications 215 (2003) 389.

[12] M. Laroche, H. Gilles, S. Girard, Optics Communications 281 (2008) 2243.

[13] D. Mallek, A. Kellou, H. Leblond, F. Sanchez, Journal of Nonlinear Optical Physics and Materials 18 (2009) 111.

[14] H. Statz, G.De Mars, Journal of Applied Physics 35 (1964) 1377.

[15] F. Sanchez, M. Le Flohic, G.M. Stephan, P. Le Boudec, P.L. François, IEEE Journal of Quantum Electronics 31 (1995) 481.

[16] F. Sanchez, G. Stephan, Physical Review E 53 (1996) 2110.

[17] A. Hideur, Etude Et Réalisation De Laser À Fibre De Puissance, University of Rouen, France, 2001, Ph.D. dissertation.

[18] N.H. Packard, J.P. Crutchfield, J.D. Farmer, R.S. Shaw, Physical Review Letters 45 (1980) 712.

[19] P. Bergé, Y. Pomeau, Ch. Vidal, L'ordre Dans Le Chaos, Hermann éditeur des sciences et des arts Paris, 1992.

[20] D. Amroun-Aliane, L. Pastur, C. Letellier, Physical Review E 83 (2011) 056212.

Fig. 9. Chaotic regime for $r=9.3003$. (a) Temporal evolution of the total output intensity and (b) first return map. 